\title{
EXIL ALS HETEROTOPIE. DIE DIMENSIONEN DER INNEREN EMIGRATION UND DES EXILS AM BEISPIEL DES LITERARISCHEN SCHAFFENS VON ERNST WIECHERT
}

\begin{abstract}
Emigration as heterotopia. Spaces and dimensions of emigration and inner emigration of Ernst Wiechert

Ernst Wiechert (1887-1950) was an East Prussian-born author who remained mentally related to the natural and cultural landscape of that region. The relation was very strongly reflected in almost all his literary work. The article deals with defining what forms and spaces of emigration and inner emigration are specific to Wiechert. It positively verifies the present state of research which treats Wiechert as the representative of inner emigration. The article also indicates new spaces of emigration (heterotopia according to Michel Foucault's definition) and inner emigration of Wiechert by careful analysis of his autobiographical texts and literary work.
\end{abstract}

KEY WORDS: Ernst Wiechert, emigration, inner emigration, heterotopia (Michel Foucault), East Prussia, landscape of East Prussia

Nach der eingehenden Erforschung einer umfangreichen Debatte, die versucht hat, Ernst Wiechert als einen Autor der Inneren Emigration (oder eben auch nicht) zu definieren, stellt sich die Frage nach der Stichhaltigkeit der Kriterien einer solchen Klassifikation, wie auch, oder vielleicht vor allem, überhaupt die Frage nach der Nützlichkeit einer solchen Klassifikation an sich. Denn letztendlich haben wir es in solch einem Fall mit subjektiven Beurteilungen in erster Linie von Biografien zu tun und nicht von literarischen Autorenbiografien, die eher Zweifel erwecken, vor allem, wenn die Autonomie des Autors berücksichtigt werden soll. Diskussionen, die im Laufe der Dekaden um den Begriff der Autonomie des Autors geführt wurden - vor allem in Zusammenhang mit der Herausgabe einer der wichtigsten Publikationen, die sich mit den Fragestellungen der Autonomie des Autors beschäftigt, nämlich der Essaysammlung Zniewolony umyst (Verführtes Denken) von Czesław 
Miłosz (1953a, deutsch 1953b) -, bestätigen nur den Standpunkt: eine Autonomie des Autors gibt es nicht. Die Schriftsteller werden nicht danach gefragt, ob sie an dem Zeitgeschehen teilnehmen möchten und ob sie bereit wären, dazu Stellung zu nehmen. Sie werden oft als Persönlichkeiten wahrgenommen, deren Pflicht es sei, eine moralische Beurteilung des Zeitgeschehens abzuliefern. Sie fühlen sich letztendlich für jene Angelegenheit verantwortlich, die Miłosz mit folgenden Worten ausdrückte, als er, der polnische Literaturnobelpreisträger des Jahres 1980, im Herbst 1989 den anderen polnischen Nobelpreisträger, jedoch für den Frieden nämlich Lech Wałęsa, damals Vorsitzender der Gewerkschaft „Solidarność“ -, in Danzig traf: „Wenn man so alt ist wie ich, dann weiß man, dass die Beseitigung der Antagonismen zwischen den Völkern wohl eines der besten Dinge ist, die man tun kann." (Lindert, 2011)

Der von Miron Białoszewski auf der Umschlagseite seines Tagebuches zum Warschauer Aufstand notierte Satz: „Nicht ich ging zur Geschichte, sie kam zu mir und überfiel mich mit Gewalt" (2016) scheint adäquat die Situation zu beschreiben, in der sich Ernst Wiechert im Kontext der Ereignisse der Zeitgeschichte während seines Lebens befunden hatte.

Auch wenn Wiechert mit den von ihm verfassten Reden, öffentlichen Schriften oder Presseartikeln, in denen es vor allem um einen moralisch-politischen Appell im Kontext der von den Nationalsozialisten geführten Politik ging, sehr oft in das öffentliche Zeitgeschehen involviert war, ist er auch oder vielleicht in erster Linie im Kontext der Literatur zu sehen.

In diesem Zusammenhang scheint es begründet zu sein, folgende Fragen zu stellen: Welche Rolle spielte für Wiechert als Menschen und Schriftsteller die von ihm in dieser politisch und historisch schweren Zeit geschaffene Literatur, und - im Hinblick darauf - um was für Formen der inneren Emigration, sowie des Exils, mit denen Ernst Wiechert definiert wurde sowie assoziiert werden kann, hat es sich gehandelt.

\section{Exil - Innere Emigration - Heterotopie}

Das Exil ist eine der radikalsten Migrationsformen und Ausdruck soziopolitischer Marginalisierung innerhalb einer Gesellschaft. Exilanten werden als Abweichler von der ideologisch, politisch und wirtschaftlich herrschenden Normgesellschaft betrachtet und erfahren ihre Marginalisierung durch Ausgrenzung und Entfremdung. Die Ausgrenzung erfolgt entweder durch die eigenen, inneren Motive oder durch von außen kommende Faktoren. Es werden, je nach einem möglicherweise gegebenen Ortswechsel, zwei Subkategorien innerhalb des Exilbegriffs unterschieden. Die erste - das innere Exil - verortet das Exil in der Heimat, die zweite - das äußere Exil - verbindet sich mit dem Aufenthalt in der Fremde. Sie werden beeinflusst von den 
Dimensionen des Ortes, des Raums und der Zeit, die nachhaltig das Exilleben zwischen Hier und Dort, zwischen Vergangenheit und Zukunft kennzeichnen. In diesem Sinne spielt sich das Leben der Exilanten im Kreuzungsbereich von Zeit und Raum $a b$, zwischen der vergangenen Zeit der verlassenen Heimat und der Gegenwart in den realen und imaginierten Orten des Exils. Den Verlust des vertrauten Lebensumfelds versuchen die Exilanten in den Orten ihres Exils zu kompensieren, indem sie sich mit Hilfe von Erinnerungen andere Orte und Räume mit alternativen Abläufen innerhalb des realen Raums und Zeitgeschehens schaffen. Sie erschaffen sich Heterotopien im Sinne von Michel Foucault (1993).

Der Begriff der Inneren Emigration entstammt der Literaturwissenschaft und entstand während der nationalsozialistischen Herrschaft in Deutschland, um die Situation eines Teils der nicht-exilierten Schriftsteller zu bezeichnen. Es war - und bleibt bis heute - ein höchst umstrittenes Konzept. Die Auseinandersetzung um die Innere Emigration und die im Exil schaffenden Schriftsteller erreichte ihren Höhepunkt in der Nachkriegszeit, als sich eine öffentliche Debatte zwischen dem in Deutschland verbliebenen Frank Thiess und dem ins Ausland Exilierten Thomas Mann entspann. Als Pendant zum äußeren Exil stellt Innere Emigration insoweit einen Typus des Widerstands gegen den Nationalsozialismus innerhalb Deutschlands dar (Grimm, 1976).

Man könnte an dieser Stelle mehrere Autoren erwähnen, die an der umfangreichen Debatte teilnahmen, unter Anderen Ralf Dahrendorf. Seine Stellungnahme scheint besonders interessant zu sein, weil er in seinem Beitrag Demokratie und Sozialstruktur (1961, S. 291) eine im Kontext dieses Beitrags aufschlussreiche These aufstellte, gegen die jedenfalls polemisiert werden kann. Dahrendorf formulierte eine eigenartige Typologie von Haltungen dem repressiven Staat gegenüber. Er unterscheidet drei Grundhaltungen: (1) kritiklose Teilnahme am politischen Geschäft, (2) Stimmenthaltung, (3) Emigration (ebd.). Man kann mit solch einer Einschätzung polemisieren, da die Innere Emigration nicht nur zum Typus (2) gerechnet werden kann, denn sie erfüllt auch die Eigenschaften des Typus (3).

Wie stark auch immer Exil und Innere Emigration voneinander unterschieden werden, sind die beiden Formen eher als ein topologischer Zustand zu betrachten. Die Unterscheidung betrifft vor allem einen Geisteszustand, einen psychologischen Zustand, in dem sich Menschen befinden, deren Emotionen und Werte auf die Trennung reagieren, wobei die Betroffenen nicht nur außerhalb, sondern auch innerhalb ihres Heimatlandes anzutreffen sind (Rodriguez, 2008a, S. 27).

Der Raum des inneren Exils steht als Raum der Gegenmacht inmitten des Machtraums, der ihn umgibt. Diese Situation erzeugt ein Spannungsfeld. Zum einen braucht der innere Exilant den Kontakt und den Zugang zum gesellschaftlichen Machtraum, um zu überleben. Zum anderen stellt der Machtraum eine Bedrohung für ihn und seine Existenz dar, vor der er sich schützen muss, indem er sich isoliert und auf eine „Reise ins Innere“ begibt. Um diesen Verlust des kulturellen Zentrums 
zu kompensieren, schaffen sich die Betroffenen alternative Orte und Räume (ebd., S. 29). Im Fall des inneren Exils sind es imaginierte Räume, die sich als Heterotopien manifestieren. Foucault definiert Heterotopien als

wirksame Orte, die in die Einrichtung der Gesellschaft hineingezeichnet sind, sozusagen Gegenplazierungen oder Widerlager, tatsächlich realisierte Utopien, in denen die wirklichen Plätze innerhalb der Kultur gleichzeitig repräsentiert, bestritten und gewendet sind, gewissermaßen Orte außerhalb aller Orte, wiewohl sie tatsächlich geortet werden können. (1993, S. 39)

Die Heterotopien können also als neu erschaffene reale oder imaginierte Räume fungieren, die den metaphorischen Verlust von Raum und Zeit zu kompensieren versuchen. ${ }^{1}$

In diesen transterritorialen Räumen existieren zugleich verschiedene parallele Zeiten: eine reale Zeit der Gegenwart, die linear fortschreitet und an den neuen realen Ort gebunden ist, sowie eine andere Zeit, die in der Vergangenheit zu dem verlorenen Zentrum gehört, immer wieder die Gegenwart durchbricht und zum Begleiter der Heterotopie wird (Rodriguez, 2008b, S. 44).

Im Hinblick auf die Grundsätze und Erscheinungsformen der Heterotopie wird hier ein Versuch gewagt, die These zu begründen, dass Wiechert mit seinem literarischen Schaffen die Anforderungen erfüllt, die es erlauben, ihn als einen Autor und Repräsentanten sowohl des inneren als auch des äußeren Exils zu definieren. Ohne die Tatsache aus den Augen zu verlieren, dass Wiechert sein ganzes Leben lang dem aktuellen politischen Geschehen gegenüber weder weltfremd noch distanziert war, muss gleichzeitig eingestanden werden, dass er mit der von ihm geschriebenen Literatur einen Raum schuf, der den metaphorischen Verlust von Raum und Zeit kompensieren sollte.

Welcher Raum und welche Zeit sind hier gemeint?

Der am 18. Mai 1887 im Forsthaus Kleinort in Ostpreußen geborene Ernst Wiechert bekannte sich sein ganzes Leben lang zu seiner Herkunft. Nicht nur in seinen fiktionalen Texten, denn Ostpreußen war Biotop fast aller seiner Romane, Erzählungen und Märchen, sondern auch in den autobiographischen Schriften.

Ich komme aus keiner „Schule“ und ich gehöre keiner Richtung an. Aber ich komme aus einer groBen Landschaft, die vieles an mir gebildet hat, und aus jener Einsamkeit, in der ein Mensch noch wachsen und werden kann. Das ganze spätere Leben hat diesen Ursprung und diese Ursprünglichkeit nicht auslöschen können, keine Bildung, keine Ratio, keine Welt. (Wiechert, 1957b, S. 739)

Wie stark ihn als Menschen und Schriftsteller Heimat und Herkunft (1957a, S. 713 f., zuerst 1932) beeinflussten, bringen nicht nur Wiecherts Aussagen zum Ausdruck, die dieser autobiographischen Skizze und den Erinnerungen Wälder und

\footnotetext{
${ }^{1}$ Es muss jedenfalls darauf hingewiesen werden, dass diese mögliche Prägung der Inneren Emigration und des Exils immerhin keine universelle und gängige Ausdruckform darstellt, die allgemein als repräsentativ für die inneren Emigranten wahrgenommen werden kann.
} 
Menschen (erschienen 1935) und Jahre und Zeiten (erschienen 1949) entstammen, denn sein ganzes literarisches Schaffen ist tatsächlich zu einer realisierten Utopie des für den Schriftsteller verlorenen ostpreußischen Lebensraumes geworden.

[...] ich [bin] ganz und gar ein Kind der Wälder. (...) Viel mußte man wissen im Walde: den Flug der Vögel und die Fährten der Tiere. Die Sprache aller Dinge, die von Gefahr redeten...Das heißt, die Sprache der Vögel, der Spinnen, der Gräser, der Fichtenzapfen, der Wolken, der Winde. Aber dann mußte ich ein Kind der Städte werden, der Begriffe, der Wissenschaften, der Dogmen, der Zivilisation. Ich trug das Kreuz wie jedermann. (1957a, S. 713 f.)

Vielleicht hatte ich schon damals den tiefen Zwiespalt zwischen Natur und Zivilisation zu ahnen begonnen, und meine Liebe zum Wald, zum Tier, zur „großen Ordnung“ würde wohl niemals das Leidenschaftliche, ja das Verzehrende ihrer Kraft erreicht haben, wenn nicht so früh mein Paradies ein verlorenes Paradies geworden wäre. (1957c, S. 85)

Der Verlust erschien für Wiechert als ein doppelter, was er erst viel später begriffen hat.

\section{Dimensionen der Inneren Emigration}

Trotz der Vielzahl an Definitionen, was der Begriff Innere Emigration zu beinhalten vermag (Gołaszewski, Kardach \& Krenzlin, 2016), und trotz sehr unterschiedlicher Standpunkte bei den Literaturwissenschaftlern, ob Ernst Wiechert dem Kreis erstrangiger Vertreter der Inneren Emigration zugerechnet werden kann, ist es nach dem gegenwärtigen Erkenntnisstand der Fachliteratur und nach eingehender Analyse der intensiven Diskussion, welche von deutschen und polnischen Literaturwissenschaftlern seit den frühen 1950er Jahren geführt wird, als vollauf gerechtfertigt anzusehen, Wiechert der Gruppe der Schriftsteller der Inneren Emigration zuzurechnen. Die Liste der Wissenschaftler, die an dieser Debatte teilnahmen, ist sehr umfangreich. 1974 legte Guido Reiner eine Dokumentation Ernst Wiechert im Dritten Reich ${ }^{2}$ vor, die eine der wichtigsten Grundlagen für die folgenden Untersuchungen über Wiechert darstellte. In diesem Kontext sind insbesondere Studien von Hildegard Chatellier (1973), Wolfgang Brekle (1985), Marcin Gołaszewski (2014), Jörg Hattwig (1984), Leonore Krenzlin (1987) und Hans-Martin Pleßke (2000) zu erwähnen.

Im Nachwort Ernst Wiechert, konservative Traditionen und Innere Emigration, das er für die polnische Ausgabe des Romans Das einfache Leben verfasst hat, stellt Hubert Orłowski (2001) fest, dass die Zuordnung einzelner Autoren zu den Reprä-

\footnotetext{
${ }^{2}$ Guido Reiner: Ernst Wiechert im Dritten Reich: eine Dokumentation; mit einem Verzeichnis der Ernst-Wiechert-Manuskripte im Haus Königsberg. Paris 1974. Der in Paris im Selbstverlag erschienene Band bildet den zweiten Teil der von Guido Reiner in vier Bänden veröffentlichten Erst-WiechertBiographie.
} 
sentanten der Inneren Emigration nicht über detektivisch genaue Beobachtungen oder die intensive Betrachtung literarischer oder kultureller Begebenheiten und deren anschließende Zuschreibung mehr oder weniger begründeter Trends aus der Vergangenheit vonstattenging, sondern über deren Definition in direkter Gegenüberstellung mit den von den Nationalsozialisten formulierten Zielen. Versteht man den Begriff „Innere Emigration“ auf diese Art und Weise, dann gehört Wiechert mit seinem Roman Das einfache Leben, mit der Legende Der weiße Büffel oder von der großen Gerechtigkeit und mit seiner Rede Der Dichter und die Zeit mit Sicherheit dieser Kategorie an. Die Breslauer Germanistin Anna Gajdis erwähnt in ihrer Monographie Entlegene Provinz. Ostpreußen in der Prosa deutscher Schriftsteller von 1866 bis 1945 (2014) weiterhin drei Stellungnahmen Ernst Wiecherts, die ihn ganz zweifellos den Autoren der „Inneren Emigration“ zurechnen lassen. Die erste war seine Rede Der Dichter und die Jugend im Jahr 1933, die zweite seine aktive Unterstützung für Pastor Martin Niemöller, der verhaftet wurde, weil er es gewagt hatte, die Annektierung Österreichs öffentlich zu kritisieren, und darüber hinaus der Bekennenden Kirche angehörte. Drittens wollte Wiechert der Annexion Österreichs selbst nicht zustimmen. Er protestierte gegen die Methoden, die bei der brutalen Unterordnung dieses Landes und seiner über Jahrhunderte gereiften Tradition und Kultur angewandt wurden. Dies alles führte letztendlich dazu, dass Wiechert von der Gestapo verhaftet und von Juli bis September 1938 im Konzentrationslager Buchenwald inhaftiert wurde.

Im Hinblick auf die Kategorisierungskriterien der Inneren Emigration scheint Wiechert jedenfalls zwei Dimensionen der Inneren Emigration zu verkörpern. Zum einen die hier schon kurz angedeutete direkte Opposition gegen die von den Nationalsozialisten formulierten Ziele. Zum anderen bringt er in den Themen und Motiven, die sein literarisches Programm kennzeichnen, den Willen zum bewussten Alleinsein und zur „Verkapselung“ zum Ausdruck, um mit Rainer Wieland (Wieland, 1995) zu sprechen.

Dieser Rückzug aber bedeutete für ihn nicht nur die Rückkehr mittels der Themen und Motive, in denen Wiechert immer wieder zu der von ihm geliebten Landschaft und zum sozialen Biotop Ostpreußens zurückkehrte, sondern er betraf auch den Akt des Schreibens selbst, den er in dem Kapitel Kleine Literaturgeschichte in seinen Erinnerungen Jahre und Zeiten sehr eingehend beschrieben hat.

Ich kam in die Stadt und ich habe erzählt wie es mir dort erging. Schon in dem Buche Wälder und Menschen. Ich nahm alles auf, mit der großen Neugier, die ich immer hatte. (...) Das meiste aber nahm ich nur mit dem Medium des Gehirns auf. Es blieb im Gehirn, und weniges erreichte das Herz. Ich wurde zwiespältig, wie es nicht zu umgehen war (...).

Ich verachtete die Welt nicht und noch weniger die Form. Ich hatte dort zu leben (...). Aber ich stand immer an der Tür, und wenn es mir zu laut wurde, schloß ich die Türe leise hinter mir. Ich konnte immer in die Stille gehen, wie ein Fisch in die Tiefe taucht. (1957b, S. 740 u. 741) 
Im Jahr 1939, kurz nach der Veröffentlichung seines Romans Das einfache Leben, verstummte Wiechert bis 1945. Diesen Roman hatte er im Frühjahr 1939 abgeschlossen, also wenige Wochen nach seiner Entlassung aus dem Konzentrationslager Buchenwald. Das einfache Leben war eine klare Absage des Schriftstellers an Krieg und Gewalt. Dass das Buch überhaupt erscheinen konnte, war nur einem Missverständnis zwischen maßgeblichen Instanzen der Kulturpolitik im „Dritten Reich", also einem Konflikt zwischen dem Propagandaministerium und dem Reichssicherheitshauptamt, zu verdanken. Das Generalthema des Buches - Flucht in den Humanismus, in die Verinnerlichung und in die Gottessuche - bestätigt die Feststellung, dass Wiechert mittels seiner Literatur eine Reise ins Innere angetreten hat. Darin kommt die zweite Dimension der Inneren Emigration, die des Rückzugs, der Vereinsamung und Isolation zum Ausdruck. Um den Verlust der aktiven Teilnahme am kulturellen Leben zu kompensieren, schafft Wiechert in seinen literarischen Texten imaginierte Räume, erdenkt Gegenplatzierungen und Widerlager, also tatsächlich realisierte Utopien, in denen die wirklichen Orte zur gleichen Zeit repräsentiert, bestritten und gewendet sind, gewissermaßen Orte außerhalb aller Orte. Dieser imaginierte Raum wird in dem dichterischen Schaffen Wiecherts durch die ostpreußische Landschaft verkörpert. Und es ist damit sowohl das kulturelle Landschaftsbild wie auch die natürliche Landschaft gemeint, in der Wiechert aufgewachsen ist und die ihn geformt hat.

Diesen imaginierten Raum verkörpert aber nicht nur die Natur und ihre oft mythologisierte Kraft, sondern auch Ostpreußens kulturelles Landschaftsbild. Für Wiecherts Identität waren die zwischenmenschlichen Beziehungen prägend. Sie treten in vielen Romanen von Wiechert hervor, stammen aus verschiedenen Phasen seines Schaffens: am meisten in den Romanen Das einfache Leben, Die Majorin und Die Jerominkinder. Der Schriftsteller legt großen Nachdruck auf die Vorzüge der dörflichen Gemeinschaft. Das Leben in der ländlich-bäuerlichen Gemeinschaft war für ihn ein optimal sozialisierendes, der Zivilisation der Stadt entgegengesetztes Soziotop. Das Dorf Sowiróg/Eulenwinkel aus dem Roman Die Jerominkinder wurde für Wiechert in den Jahren von 1940 bis 1945, also in der Zeit, in der er sich aus dem öffentlichen Leben zurückgezogen und an dem Roman geschrieben hat, zu einem imaginierten Raum, einer tatsächlich realisierten Utopie: zu seiner Heterotopie also.

\footnotetext{
Vor einem Jahr habe ich den zweiten Band der Jeromin-Kinder geschrieben. Ich würde es nicht gewagt haben, wenn Picard mich nicht immer wieder dazu ermahnt hätte. (...) Es war mir als hätte ich mein ganzes Leben aufgezeichnet, (...) Aber mir ist, als wäre ich immer noch da, unter den Menschen von Sowirog, und als sähe auch ich das fahle Pferd im Nebel der Moore verschwinden. (1957b, S. 735)
}

Das Dorf Sowiróg/Eulenwinkel verkörperte eine ursprüngliche menschliche Gemeinschaft, die auf Werten wie Zugehörigkeit und unmittelbarem Kontakt beruht. Es war - um hier die Theorie des Kulturanthropologen Robert Redfield zu bemühen 
- eine folk society, die sich durch die starke Gruppensolidarität der Urgesellschaft auszeichnet. Dabei handelt es sich um eine relativ kleine Gruppe, die über ein bestimmtes Gebiet verstreut war; die Angehörigen dieser Gruppe kannten sich, und sie hatten das Gefühl, dass sie einem ganz bestimmten Winkel der Erde entstammen einem Land, das schon immer ihr Eigentum war, das ihr Eigentum ist und es stets sein wird (Redfield, 1962, S. 237).

Wiechert lebte in seiner Welt, in dem von ihm geschaffenen Raum, dem er das Überleben in jener Zeit verdankt. Diesen Raum schuf Wiechert unter Rückgriff auf den deutschen Idealismus und ausgehend von seinem eigenen festen Glauben an den deutschen Geist. Es lässt sich annehmen, dass auf diesem idealistischen, romantischen, nach innen gekehrten Wesenszug des deutschen Geistes Wiecherts Enttäuschung und letztendlich Desinteresse an den äußeren, den gesellschaftlichen und politischen Vorgängen des Daseins beruhten, die er in den letzten zwei Jahren seines Lebens verachtete. Damit gab Wiechert der Öffentlichkeit zu verstehen, dass das vermeintlich wahre Leben einer Nation sich auf einer anderen Ebene, eben jener des Geistes, des Intellekts, der Seele vollziehe (Sontheimer, 1982).

Sowohl diese Flucht in das Reich der Verinnerlichung als auch das Schaffen einer irrealen Wirklichkeit in der ostpreußischen Provinz wurden Wiechert oft vorgeworfen - nicht zuletzt von eifernden Nationalsozialisten: „Ernst Wiecherts geistige Struktur ist nicht die eines gesunden Menschen (...). Im Blutstrom seiner Herkunft rauscht die Nacht slawischer Schwermut (...). Die Sehnsucht nach den unendlichen Wäldern (...) wird zur kranken Kraft seines Dichtertums" (Gstettner, 1936).

Dass Wiechert missverstanden oder gar nicht verstanden wurde, lag eben auch an seiner Herkunft und seiner Prägung durch Masuren (dem Masurentum, poln. Mazurskość). Das Land seiner Herkunft - Ostpreußen, zu dem er sich Zeit seines Lebens bekannte - wurde von der breiten Öffentlichkeit, aber auch von den Gebildeten bloß als Peripherie des Deutschtums wahrgenommen, obwohl ihm viele anerkannte Persönlichkeiten entstammten, darunter als die Größten J. G. Herder und I. Kant.

Die hier erwähnten Ereignisse aus Wiecherts Leben, die von seinem Heroismus zeugen, wenn es um seine Einstellung zum Nationalsozialismus geht, platzieren ihn im Kreis der Schriftsteller der Inneren Emigration; aber auch die zahlreichen literaturwissenschaftlichen Analysen (Roslan, 1992; Schnell, 1967; Ebeling, 1937 u. 1947; Chojnowski, 2011; Gajdis, 2014), in denen Beispiele angeführt werden, wie Wiechert seine literarischen Figuren zeichnet und ihre Entscheidungen formuliert, sind ein Beweis für einen entschiedenen Widerstand gegen die nationalsozialistische Ideologie. In diesem Kontext darf nicht verschwiegen werden, dass Wiecherts literarisches Debüt mit den Romanen Der Wald (erschienen 1922) und Der Totenwolf (erschienen 1924) ihn dem Schriftstellerkreis, den Vertretern der nationalistischen oder sogar völkischen Ideen zurechnen ließ, was ihn bis an sein Lebensende belastete, obwohl er sich Ende 1920er Jahre von der Weltanschauung der rückwärtsge- 
wandten Revolutionäre entschieden distanziert hatte. Diesen Wandel in seinem Leben bezeichnete Wiechert selbst als einen Gnadenumbruch (Pleßke, 1988, S. 763). In allen seinen späteren literarischen Werken wahrt er den politischen Ereignissen gegenüber Abstand.

Wiecherts Schriftstellerexistenz als Lebensweg eines Menschen - die ihn umgebende Realität also - und der Gehalt seiner Romane - die Fiktion als ein imaginierter Ort, als ein anderer Ort und Raum mit alternativen Abläufen innerhalb des realen Raums und Zeitgeschehens - bilden zwei Ebenen des Exilbegriffs - einer in der Heimat sich abspielenden Inneren Emigration.

Es lässt sich aber noch eine weitere Dimension von Wiecherts Innerer Emigration feststellen. Sie erfolgt im Akt des Schreibens. Er befreite Wiechert von dem real existierenden Raum und dem Zeitgeschehen. Der Akt des Schreibens lässt sich auch im Fall Wiecherts ebenso als eine von ihm geschaffene Heterotopie bezeichnen. Dies wird augenfällig in der Sprache, deren sich der Autor in seinen Romanen bedient. Er wird als Dichter der Stille bezeichnet, der jedoch auf die Kraft des Wortes setzt (Pleßke, 2000, S. 109). Das Lesen ist mit dem Genuss seiner wohlklingenden, an Metaphern reichen Sprache verbunden. Gleichzeitig kann man die Selbstberauschung des Autors an dem spüren, was die Figuren im erzählten Text in ihren Äußerungen von sich geben, denn man fühlt, dass die Figuren daran „Spaß“ haben, und dem Leser teilt sich ohne Weiteres diese Selbstberauschung der Figuren mit. Es fehlte aber nicht an vielen Stimmen, die an seinem literarischen Ton Kritik übten, er sei zu pathetisch, zu gehoben, zu künstlich, nicht der Wirklichkeit angemessen (Kardach \& Gołaszewski, 2016, S. 86). Als Dichter befand sich Wiechert deshalb auf gleichsam exterritorialem Gebiet, was er eigentlich selbst schon 1936 in Wälder und Menschen zu bestätigen vermochte:

Eingebettet in die grenzenlosen Wälder, in den Lauf der Jahreszeiten, in die Liebe einer kleinen Gemeinschaft, früh dem Leid und den Träumen hingegeben, früher Erschütterung fähig, fromm und noch sündenlos. Aber alles schon leise beschattet von einer gegenstandslosen Sehnsucht, dem Alltag nicht immer gewachsen, kein Held und kein Eroberer, mehr betrachtend als tätig, früh geneigt, Besonderes zu verklären und vor dem Wirklichen in das Unwirkliche zu flüchten. (1957c, S. 29)

Wiechert bestätigt jedenfalls in seinen autobiographischen Texten, dass eben der Akt des Schreibens selbst ihn zu retten vermochte, dass er seine Zuflucht war. Auf folgende Art und Weise beschrieb er dies in einer Aussage über seinen Roman Das einfache Leben:

Für die anderen war es ein Buch wie andere Bücher, nur noch stiller, noch innerlicher, und ich wußte damals noch nicht, welch ein Trost es für Unzählige werden würde. Für mich aber, als ich es schrieb, war es mehr. Es war „mein“ Buch, das einzige meiner Bücher vielleicht, das ganz mein war. Es war nicht nur die Flucht vor den Eumeniden, es war der Sieg über sie. Es war ein Traumbuch, in dem ich mich mit Flügeln über diese grauenvolle Erde hinaushob. Mit ihm spülte ich mir 
von der Seele, was sie beschmutzt, befleckt, erniedrigt, entwürdigt und zu Tode gequält hat. Mit ihm gingen die Schatten und die Toten fort, nicht in das wesenlose Nichts, sondern in ein beglänztes Land der Erinnerung und der Verklärung. Mit ihm baute ich noch einmal eine Welt auf, nachdem die irdische mir zusammengebrochen oder schrecklich entstellt worden war. Nicht eine wirkliche, aber eine mögliche, und jede mögliche Welt ist auch eine wahre Welt. (1957b, S. 688 f.)

\section{Dimensionen des Exils}

Anschließend ein vielleicht wagemutiges Unterfangen, das den Ortswechsel ins Exil thematisiert - den Weg in die Fremde, auf dem Wiechert als der äußere Exilant im eigenen Land erscheint.

1930 zog Wiechert von Königsberg nach Berlin um, drei Jahre später ließ er sich in Ambach am Starnberger See nieder, und 1936 fand er auf dem oberbayerischen Gagert-Hof sein neues Zuhause, wo er bis 1948 blieb. Die letzten beiden Jahre seines Lebens verbrachte er in der Schweiz, auf dem Rütihof. Seine ostpreußische Heimat besuchte er zum letzten Mal im Sommer 1936. Danach sah sich Wiechert gezwungen, neue Räume zu erobern, sich eigene, transterritoriale Platzierungen zu schaffen, in welchen er versuchte, das verlorene Zentrum als Heterotopie in neuen und realen fremden Räumen wiederherzustellen. In diesen Räumen existierten für ihn zugleich parallele Zeiten: eine reale Zeit der Gegenwart, die linear fortschreitet und an den neuen, realen Ort gebunden ist, sowie eine alternative Zeit, in der die Vergangenheit zu dem verlorenen Zentrum gehört, immer wieder die Gegenwart durchbricht.

Ein Textabschnitt, der diesen Zustand zu bestätigen vermag, stammt aus der kurzen autobiographischen Erzählung In der Heimat:

\footnotetext{
Als ich in mein fünfzigstes Lebensjahr ging, hat meine Heimat mich zum letzten Mal gerufen. Mein Vater rief mich. (...) So machte ich mich denn auf den Weg. Schön war das Land unter Sonne und Gewitterwand. Das Korn reifte, und der Wind schmeckte nach Brot. Aber es war mir alles nun Fremde, Bayern oder das Frankenland oder der Thüringer Wald. Es war mir, als sitze mein Vater die ganze Zeit an seinem Fensterplatz und sehe mir entgegen wie die helle Strasse unter den jagenden Reifen zurückflog, die weißen Birkenstämme, die alten Meilensteine. Als sei das alles Fremde, unvermeidlicher Raum, ein Irrtum des Lebens, (...) und die Wirklichkeit werde erst beginnen, wenn der Wind meiner Kindheit mir wieder entgegenkommen werde. Ich sah die Straßen, die Städte, die Brücken, aber ich sah sie nur als einen Vordergrund, der sich verschob und zurückblieb, und das einzig Unverrückbare, das Ziel und das Ende waren jenes kleines Haus und der alte Mann, der auf mich wartete, und der große Wald, der auf uns beide wartete. (1993, S. 14-16) (Hervorhebungen M.K.)
}

Ende 1936 starb Ernst Wiecherts Vater. Der Schriftsteller wohnte damals schon auf dem Gagert-Hof. Er erinnert sich an diesen Tag:

Sie schrieben mir, daß er gestern gestorben war, mit einem hellen Schein auf seinem müden Gesicht. (...) Ich bekam diese Nachricht an einem klaren Wintertag, vor der Abendzeit. Die Sonne 
wollte untergehen, es lag viel Schnee, und ich ging aus meinem Tor, weit in die Wälder hinein. (...) Ich stand vor dem Moor, das so aussieht, als könnte es auch in der Heimat liegen. Das Land war fremd (...). (ebd., S. 32 u. 34) (Hervorhebung M.K.)

Die ostpreußische Landschaft, zu der für Wiechert als ein unentbehrliches Element der Wald gehört, wird für ihn, den Exilanten, nicht bloß zu einem anthropologischen Ort, sondern zu einem Ort der Erinnerung, der nicht nur identitätsstiftend ist, sondern die Identität geradezu aufrechterhalten soll. Wiechert versuchte, das verlorene Zentrum als Heterotopie in neuen und realen fremden Räumen wiederherzustellen, auch wenn es um alltägliche Arbeiten auf dem Gagert-Hof ging:

Wir haben einen kleinen Wald gepflanzt, auf dem Hang unter den alten Eichen hinter unserem Hause. Ein heißer Wind des letzten Jahres hat ihn versengt, aber wir haben ihn neu gepflanzt. Er wird uns keinen Schatten mehr geben, aber ich will im Kleinen tun, was meine Väter getan haben. (ebd., S. 36)

In Erinnerung an die ersten Tage nach dem Ende des Zweiten Weltkrieges schrieb er in Jahre und Zeiten:

Es mag für einen anderen, diesseits oder jenseits der Grenze, nicht leicht sein, ohne Tadel oder Bitterkeit zu begreifen, wie jemand, der sein Land und seine Sprache liebt wie ich, vom ersten Tage des Krieges an nichts anderes konnte, als um den Sieg der Feinde beten. Aber er übersieht wohl, daß der Sieg des sogenannten Vaterlandes hier mehr gewesen wäre als ein Sieg der Waffen und eine Stärkung des Raumes, in dem unsere Sprache gesprochen wurde. Mehr als der Sieg der Waffen, weil es ein Sieg der Gewalt, des Bösen und Unmenschlichen gewesen wäre. Mehr als eine Stärkung des deutschen Raumes, weil es eine Unterjochung und Versklavung der halben Erde bedeutet hätte. Auf einer solchen Erde zu leben, würde nicht nur für uns unmöglich geworden sein, es würde die Wurzeln aller durchschnitten haben, die im Guten, Rechten und Wahren zu leben gewillt waren und anders nicht leben konnten. Von einer solchen Erde gab es nur die Flucht in einen ihrer unberührten Teile oder in den Tod.

(...)

$\mathrm{Ob}$ es nicht ein Eigensinn des Abseitigen sei, eine Verdunkelung und Verhärtung des Urteils und ob das Leben selbst, die Tatsachen, die Ereignisse ihn nicht zur Umkehr aufriefen. Dann pflegte ich in den Wald zu gehen als in die alte Heimat meiner Seele und dort über dem Moor zu sitzen, lange Zeit, meine Hände in das Gras zu stützen und hinausblicken in das grüne Schweigen bis zu dem weiten Horizont, wo ein Kirchturm sich in die blaue Luft emporhob oder ein fernes Gespann, winzig vor dem großen Himmelsgewölbe, den Pflug hinter sich her durch den Acker zog. Und jedesmal wurde es wieder ruhig in mir. (1957b, S. 700 u. 702) (Hervorhebungen M. K.)

Ernst Wiechert wandelte Zeit seines Lebens an der Grenze von zwei Welten, der realen und einer verklärten, imaginären Welt der Erinnerung. In den letzten Erinnerungen gestand er:

Ich hatte das Glück, daß zu Beginn meines Lebens nur große Dinge um mich standen und daß sie große Schatten warfen. Nicht ein Hinterhaus, eine Mietwohnung, eine gepflasterte Erde. Sondern daß der Wald da war, ein großer Wald, große Wolken an einem großen Himmel, und es war etwas wie Unendlichkeit darin. 
Das war meine Welt ... Ich wuchs nicht in einem Café auf wie die Literaten. Ich lernte das alles kennen, viel später, aber ich lernte es kennen wie Brehms Tierleben. Hinter den Bildern und Ordnungen und Beschreibungen blieb immer die Welt des Ursprungs stehen. Das andere war das Durchsichtige, diese war das Dichte und Undurchsichtige. (ebd., S. 739 u. 740)

Zum Abschluss meiner Überlegungen zur Emigration als Heterotopie, mithin zu einem - ich zögere nicht, dies festzustellen - Raum nicht nur der Emigration, sondern auch einem zum Überstehen dieser Emigration geschaffenen Raum, sollte im Falle Wiecherts, für den diesen Raum in hohem Maße die ostpreußische Landschaft darstellte, das Spezifische der von diesem Autor kreierten Landschaft in den Kontext des breiteren Diskurses über die ostpreußische Landschaft gestellt werden.

In seiner Monografie Wschodniopruskość, die das Phänomen einer durch verschiedene Determinanten geschaffenen ostpreußischen Regionalidentität untersucht, widmet Robert Traba ein ganzes Kapitel dem Einfluss der ostpreußischen Landschaft auf die Herausbildung einer kollektiven Identität der Ostpreußen (2005, S. 218-236). Dabei lenkt er das Augenmerk auf die Verbindung von Natur- und Kulturlandschaft, die im Falle der Provinz Ostpreußen besonderen Einfluss auf die Verwurzelung ihrer Bewohner im Raum und die Entstehung eines Zugehörigkeitsgefühls zu dieser Region hatte. Traba nennt folgende Vermittlungswege zwischen Landschaft und Regionalidentität: Bildung, Reiseführer und Literatur. Aus der Analyse des Phänomens der ostpreußischen Landschaft in den verschiedenen Aspekten ihrer öffentlichen Rezeption zieht Traba ähnliche Schlüsse wie der Posener Germanist Hubert Orłowski, der dieses Problem in einer Reihe von Veröffentlichungen untersucht hat $(2003,2007,1994,2004)$ und die Landschaft der ,dunklen Wälder und kristallnen Seen" als eine der wichtigsten Determinanten für die ostpreußische Regionalidentität ansieht. In der nach dem Ersten Weltkrieg geschaffenen Literatur von mit Ostpreußen verbundenen Schriftstellern spiegelt sich nicht nur die Faszination für diese Landschaft wider, sondern auch die Erstellung eines Katalogs von Ikonen und Landschaftsveduten, die von den Propagatoren einer ostpreußischen Identität als Kanon von Identifikationsmustern mit Ostpreußen angesehen wurden.

Das herausragendste ethnisch-regional-nationale Identifikationsmuster beziehungswiese -feld der Ostpreußen-Literatur heißt die Landschaft, und es nimmt einen nicht zu überschätzenden Standort ein. (...) Für das literarische Bild Ostpreußens nach dem Ersten Weltkrieg hingegen ließe sich ein Katalog aufstellen, ein Kultur-Alphabet, mit stimmungsvollen, leitmotivisch auftretenden Gedächtnisorten, Figurationen und Details. Der signifikante Kanon beinhaltet unter anderem solche Ikonen wie: die Kurische Nehrung, das Frische Haff, die Düne und den Bernstein, den Wald und den See, die Rominter Heide und das Pferdegestüt Trakehnen, den Elch und den Kranich, Königsberg und die Marienburg. (Orłowski, 2003, S. 271)

Der erwähnten Faszination kam bei der Bildung eines ostpreußischen Regionalbewusstseins eine besondere Rolle zu, wobei sie erst nach dem Ersten Weltkrieg zu einer nationalen oder auch regionalen Determinante wurde, als die „vorideologische 
Phase der ostpreußischen Landschaft“ beendet war und sie in den Rang einer „,spezifisch deutschen Landschaft" erhoben wurde. Die Verkünder einer ostpreußischen Identität betrachteten die genannten Ikonen als Teil eines ,planmäßigen und bewussten Handelns der nationalen Gemeinschaft", das dieser Landschaft eine „,deutsche Seele" verschaffen sollte (Orłowski, 2008, S. 107 f.).

Ganz anders verhält es sich jedoch mit der literarischen Kreation der Landschaft in Wiecherts Schaffen. Das „Ostpreußentum“ der Landschaft in Wiecherts literarischen Werken liegt jenseits des Diskurses, der der Landschaft eine nationale identitätsbildende Funktion zuweist. Allerdings lässt sich die Wiechertsche Kreation der Landschaft, bei der die Elemente der natürlichen Landschaft wie Wald, See oder Scholle einem intensiven Mythologisierungsprozess unterzogen werden, als Determinante einer regionalen Identität ansehen, wobei die Natur hier eher die Rolle eines eigentümlichen, sicheren Unterschlupfes spielt, oder gar einer Zuflucht vor den Unbilden der Geschichte (Kriege, Ideologien), den „Errungenschaften“ der Zivilisation, der Modernisierung oder auch bei persönlichen Problemen. Letztendlich ist der „Wiechertsche Wald“ auch ein besonderes Gegenmittel gegen die nationalsozialistische Ideologie, was zugleich auf ein weiteres Element in Wiecherts literarischem Schaffen hinweist, das seine Stellung im Kreise der Schriftsteller der Inneren Emigration untermauert.

\section{Bibliographie}

Białoszewski, M. (2014). Das geheime Tagebuch. Berlin: edition.fotoTAPETA.

Białoszewski, M. (2016). Pamiętnik z powstania warszawskiego. Warszawa: Państwowy Instytut Wydawniczy.

Brekle, W. (1985). Schriftsteller im antifaschistischen Widerstand 1933-1945 in Deutschland. Berlin: Aufbau.

Chatelier, H. (1973). Ernst Wiechert im Urteil der deutschen Zeitschriftenpresse 1933-1945. Recherches germaniques, 153-195.

Chojnowski, Z. (2011). Od biografii do recepcji. Ernst Wiechert, Konstanty I. Gałczyński, Zbigniew Herbert na Warmii i Mazurach. Olsztyn: Wydawnictwo Uniwersytetu Warmińsko-Mazurskiego.

Dahrendorf, R. (1961). Gesellschaft und Freiheit. München: Piper.

Ebeling, H. (1937). Ernst Wiechert. Der Weg eines Dichters. Berlin: Grote.

Ebeling, H. (1947). Ernst Wiechert. Das Werk des Dichters. Wiesbaden: Limes.

Foucault, M. (1993). Andere Räume. In K. Barck, P. Gente, H. Paris \& S. Richter (Hrsg.), Aisthesis: Wahrnehmung heute oder Perspektiven einer anderen Ästhetik. Leipzig: Reclam.

Gajdis, A. (2014). Odległa prowincja. Prusy Wschodnie w prozie pisarzy niemieckich (1866-1945) [Entlegene Provinz. Ostpreußen in der Prosa deutscher Schriftsteller von 1866 bis 1945]. Wrocław: Atut.

Gołaszewski, M. (2014). Ernst Wiechert und sein Werk im Spiegel des autobiographischen Werkes Jahre und Zeiten. Der literarische Werdegang eines deutschen Dichters der Inneren Emigration. Vom Totenwolf (1924) über Totenwald (1939/1946) bis Missa sine nomine (1950). In J. Płuciennik (Hrsg.), Zagadnienia Rodzajów Literackich. Problems of Literary Genres (S. 185-211). Łódź: Instytut Kultury Współczesnej. 
Gołaszewski, M., Kardach, M. \& Krenzlin L. (Hrsg.). (2016). Zwischen Innerer Emigration und Exil. Deutschsprachige Schriftsteller 1933-1945. Berlin: Gruyter.

Grimm, R. (27. September 1976). Innere Emigration als Lebensform. In R. Grimm \& J. Hermand (Hrsg.), Exil und Innere Emigration. Frankfurt a. M..: Athenäum.

Gstettner, H. (1936). Ernst Wiechert und die Jugend. Völkischer Beobachter. Württembergische Ausgabe, Nr. 271.

Hattwig, J. (1984). Das dritte Reich im Werk Ernst Wiecherts. Frankfurt a. M.: Lang.

Kardach, M. \& Gołaszewski, M. (2017). Macht und Kultur unter dem Einfluss der Kulturpolitik vor und nach 1945 am Beispiel des literarischen Werkes von Ernst Wiechert. In D. Haberland (Hrsg.), Ästhetik und Ideologie 1945. Wandlung oder kontinuität poetologischer Paradigmen deutschsprachiger Schriftsteller (S. 77-88). München: De Gruyter Oldenbourg.

Krenzlin, L. (1987). Suche nach einer veränderten Lebenshaltung. Ernst Wiechert: „Das einfache Leben“. In S. Bock \& M. Hahn (Hrsg.), Erfahrung Nazideutschland. Romane in Deutschland 1933-1945 (S. 384-411). Berlin: Aufbau.

Lindert, C. (2011). „Verführtes Denken“ machte ihn weltberühmt. Deutschlandfunk, abgerufen von http://www.deutschlandfunk.de/verfuehrtes-denken-machte-ihn-weltberuehmt.871.de.html?dram: article_id=127380 am 26. Oktober 2016.

Miłosz, Cz. (1953a). Zniewolony umyst. Paris: Instytut Literacki.

Miłosz, Cz. (1953b). Verführtes Denken. Köln: Kiepenheuer \& Witsch.

Orłowski, H. (1994). Literatura peryferii czy pogranicza? Prusy Wschodnie w powojennej literaturze niemieckiej. Komunikaty Mazursko-Warmińskie, 1 (203), 43-52.

Orłowski, H. (2001). Posłowie. Ernst Wiechert a tradycje konserwatywne i emigracja wewnętrzna. In E. Wiechert: Proste życie (S. 339-359). Olsztyn: Borussia.

Orłowski, H. (2003). Za górami za lasami... O niemieckiej literaturze Prus Wschodnich 1863-1945. Olsztyn: Borussia.

Orłowski, H. (2003). Das Bild Ostpreußens in der deutschen Literatur des 20. Jahrhunderts. In M. Weber (Hrsg.), Preußen in Ostmitteleuropa. Geschehensgeschichte und Verstehensgeschichte (S. 259-281). München: Oldenbourg.

Orłowski, H. (2007). Hinter den sieben Bergen... Zur ostpreußischen Literatur 1863-1945. Dresden: w.e.b.

Orłowski, H. (2008). Ostpreußen. Wiederentdeckung einer historischen Provinz? Regionalismus und Literaturgeschichtsschreibung. Kwartalnik Neofilologiczny, 55, H. 2, 105-119.

Orłowski, H. (2004). Landschaft als Blickfang. Von der Erfindung der Provinz Ostpreußen. In M. K. Lasatowicz (Hrsg.), Kulturraumforschung. Sprachpolitische, kulturpolitische, ästhetische Dimensionen (S. 225-233). Berlin: Trafo.

Pleßke, H.-M. (1988). Vom Wort als Macht des Herzens. Versuch über Ernst Wiechert. Sinn und Form, 40, 763.

Pleßke, H.-M. (2000). Verteidiger der gefährdeten Menschentums. Über Ernst Wiechert. In F.-L. Kroll (Hrsg.), Deutsche Autoren des Ostens als Gegner und Opfer des Nationalsozialismus. Beiträge zur Widerstandsproblematik (S. 92-109). Berlin: Duncker \& Humblot.

Redfield, R. (1962). The Folk Society. In M. P. Redfield (Hrsg.), Human Nature and the Study of Society. The Papers of Robert Redfield. Chicago: University Press.

Reiner, G. (1974). Ernst Wiechert im Dritten Reich. Eine Dokumentation; mit einem Verzeichnis der Ernst-Wiechert-Manuskripte im Haus Königsberg. Paris: Selbstverlag.

Rodriguez, F. A. (2008). Exil als Heterotopie. Kubanische Kunst am Ende des 20. Jahrhunderts. Frankfurt a. M.: Lang.

Rodriguez, F. A. (2008). Ein Interview mit J. M. Pozo im Künstleratelier in Berlin (unveröffentlicht). 
Rosłan, J. (1992). Ernst Wiechert. Życie i dzieło. Olsztyn: Ośrodek Badań Naukowych.

Schnell, R. (1967). Literarische innere Emigration 1933-1945. Stuttgart: Metzler.

Sontheimer, K. (1982). „Der deutsche Geist” - eine Tradition ohne Zukunft. Merkur 36, 3, 32-243.

Traba, R. (2005). Wschodniopruskość. Tożsamość regionalna i narodowa w kulturze politycznej Niemiec (S. 218-236). Poznań: Towarzystwo Przyjaciół Nauk.

Wiechert, E. (1957a). Heimat und Herkunft. In Sämtliche Werke in zehn Bänden, Bd. 10 (S. 712-714) München: Desch.

Wiechert, E. (1957b). Jahre und Zeiten. Erinnerungen. In Sämtliche Werke in zehn Bänden, Bd. 9 (S. 331-800). München: Desch.

Wiechert, E. (1957c). Wälder und Menschen. Eine Jugend. In Sämtliche Werke in zehn Bänden, Bd. 9 (S. 5-196). München: Desch.

Wiechert, E. (1993). W rodzinnych stronach. In der Heimat (Übersetzung: M. Podlasek-Ziegler, Vorwort: B. Ollech). Olsztyn: Borussia.

Wieland, R. (1995). „ein perpetuum mobile von schmerzen?“ Fragen an eine vergleichende Exilwissenschaft. In H. Haarmann (Hrsg.), Innen-Leben: Ansichten aus dem Exil (S. 151-162). Berlin: Fannei und Walz. 